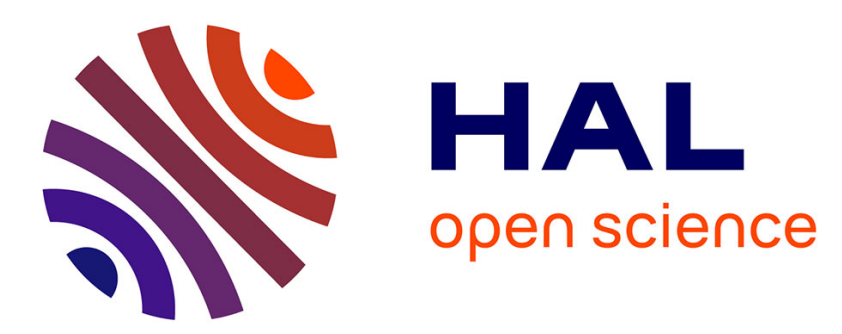

\title{
$\beta$ phase instability in poly(vinylidene fluoride/trifluoroethylene) thin films near $\beta$ relaxation temperature
}

B.B. Tian, X. F. Bai, Y. Liu, P Gemeiner, X. L. Zhao, B. L. Liu, Y.H. Zou, X.D. Wang, H. Huang, J.L. Wang, et al.

\section{To cite this version:}

B.B. Tian, X. F. Bai, Y. Liu, P Gemeiner, X. L. Zhao, et al.. $\beta$ phase instability in poly(vinylidene fluoride/trifluoroethylene) thin films near $\beta$ relaxation temperature. Applied Physics Letters, 2015, 106 (106), pp.092902. 10.1063/1.4913968 . hal-01258698

\section{HAL Id: hal-01258698 \\ https://hal.science/hal-01258698}

Submitted on 24 Aug 2020

HAL is a multi-disciplinary open access archive for the deposit and dissemination of scientific research documents, whether they are published or not. The documents may come from teaching and research institutions in France or abroad, or from public or private research centers.
L'archive ouverte pluridisciplinaire HAL, est destinée au dépôt et à la diffusion de documents scientifiques de niveau recherche, publiés ou non, émanant des établissements d'enseignement et de recherche français ou étrangers, des laboratoires publics ou privés. 


\section{$\beta$ phase instability in poly(vinylidene fluoride/trifluoroethylene) thin films near $\beta$ relaxation temperature}

Cite as: Appl. Phys. Lett. 106, 092902 (2015); https://doi.org/10.1063/1.4913968

Submitted: 30 December 2014 . Accepted: 19 February 2015. Published Online: 03 March 2015

B. B. Tian, X. F. Bai, Y. Liu, P. Gemeiner, X. L. Zhao, B. L. Liu, Y. H. Zou, X. D. Wang, H. Huang, J. L. Wang, Sh. Sun, J. L. Sun, B. Dkhil, X. J. Meng, and J. H. Chu
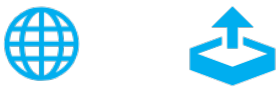

\section{ARTICLES YOU MAY BE INTERESTED IN}

The creep process of the domain switching in poly(vinylidene fluoride-trifluoroethylene) ferroelectric thin films

Applied Physics Letters 103, 042909 (2013); https://doi.org/10.1063/1.4816749

Abnormal polarization switching of relaxor terpolymer films at low temperatures

Applied Physics Letters 102, 072906 (2013); https://doi.org/10.1063/1.4793188

Temperature dependence of elastic, dielectric, and piezoelectric properties of "single crystalline" films of vinylidene fluoride trifluoroethylene copolymer

Journal of Applied Physics 81, 2760 (1997); https://doi.org/10.1063/1.364300

\section{Lock-in Amplifiers up to $600 \mathrm{MHz}$}
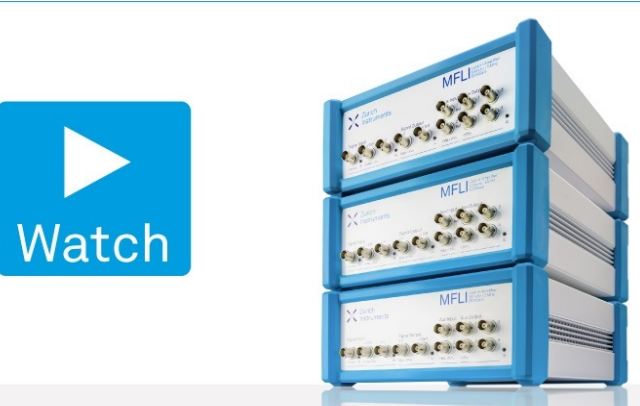


\title{
$\beta$ phase instability in poly(vinylidene fluoride/trifluoroethylene) thin films near $\beta$ relaxation temperature
}

\author{
B. B. Tian, ${ }^{1,2,3}$ X. F. Bai, ${ }^{2}$ Y. Liu, ${ }^{2}$ P. Gemeiner, ${ }^{2}$ X. L. Zhao, ${ }^{1,3}$ B. L. Liu, ${ }^{1,3}$ Y. H. Zou, ${ }^{1,3}$ \\ X. D. Wang, ${ }^{1}$ H. Huang, ${ }^{1,3}$ J. L. Wang, ${ }^{1,3, a)}$ Sh. Sun, ${ }^{1,3}$ J. L. Sun, ${ }^{1,3}$ B. Dkhil, ${ }^{2, b)}$ \\ X. J. Meng, ${ }^{1,3}$ and J. H. Chu ${ }^{1,3}$ \\ ${ }^{1}$ National Laboratory for Infrared Physics, Shanghai Institute of Technical Physics, Chinese Academy \\ of Sciences, Yu Tian Road 500, Shanghai 200083, China \\ ${ }^{2}$ Laboratoire Structures, Propriétés et Modélisation des Solides (SPMS), CentraleSupélec, UMR-8580 CNRS, \\ Université Paris-Saclay, F-92290 Châtenay-Malabry, France \\ ${ }^{3}$ University of Chinese Academy of Sciences, No. 19A Yuquan Road, Beijing 100049, China
}

(Received 30 December 2014; accepted 19 February 2015; published online 3 March 2015)

\begin{abstract}
The $\beta$ phase stability in poly(vinylidene fluoride/trifluoroethylene) $[\mathrm{P}(\mathrm{VDF}-\mathrm{TrFE})]$ thin films was studied below $300 \mathrm{~K}$ using X-ray diffraction and polarization-electric-field $(P-E)$ hysteresis loops measurements. On as-grown samples, an irreversible partial order-disorder transformation at $T_{\beta} \sim 250 \mathrm{~K}$, namely, the $\beta$ relaxation temperature, was evidenced by the appearance of an additional X-Ray diffraction peak above $T_{\beta}$ as well as changes on the $P$-E loops on heating after the first cooling. This order-disorder-like transformation which is attributed to an all-trans order to helical disorder transition is suggested to take place in defect-rich regions like crystal-amorphous interphases and/or crystalline areas with randomly distributed TrFE defect-like units. (C) 2015 AIP Publishing LLC.

[http://dx.doi.org/10.1063/1.4913968]
\end{abstract}

Poly(vinylidene fluoride/trifluoroethylene) P(VDF-TrFE) copolymers are typical ferroelectrics and have attracted great attention for their good ferroelectricity and some unique features, like flexibility and self-healing. ${ }^{1-4}$ The P(VDF-TrFE) polymers are considered as interesting candidates for development of many electronic devices such as smart labels, rollable displays, contactless identification transponders, and actuators in Braille displays. ${ }^{5-8}$ Unlike pure poly(vinylidene fluoride) (PVDF), P(VDF-TrFE) copolymers exhibit a Curie temperature $\left(T_{c}\right)$ related to the phase transition which is below the melting temperature and increases with the VDF mol. \%. ${ }^{9}$ $\mathrm{P}(\mathrm{VDF}-\mathrm{TrFE})$ copolymers are thus in their ferroelectric phase and present ideal polarization switching current at room temperature. ${ }^{10}$ This ferroelectric phase has the same structure as the $\beta$ phase of pure PVDF with a slightly expanded unit cell, which is characterized by a pseudo-hexagonal packing of parallel chains in the extended trans-trans conformation. ${ }^{11,12}$ According to the symmetry of the $C m 2 m$ orthorhombic space group, the molecular dipole moments (perpendicular to the chain axis) are aligned parallel to the longitudinal mirror plane of the chains. ${ }^{13}$ Note that this space group represents only an average symmetry of $\mathrm{P}(\mathrm{VDF}-\mathrm{TrFE})$ copolymers because there is existence of statistically random substitution of VDF monomers by TrFE monomers. ${ }^{14}$ The good ferroelectric, pyroelectric, and piezoelectric properties are related to the $\beta$ phase and thus its stability in $\mathrm{P}(\mathrm{VDF}-\mathrm{TrFE})$ copolymers is of crucial importance.

In the present study, the thermal stability of $\beta$ phase in $\mathrm{P}(\mathrm{VDF}-\mathrm{TrFE})(70 / 30 \mathrm{~mol}$. \%) thin films was investigated using X-ray diffraction from $100 \mathrm{~K}$ to $300 \mathrm{~K}$. The P(VDFTrFE) thin films were deposited on Au-coated silicon substrates by spin-coating method. The film thickness determined

\footnotetext{
${ }^{\text {a)} E-m a i l: ~ j l w a n g @ m a i l . s i t p . a c . c n ~}$

b)E-mail: brahim.dkhil@ecp.fr
}

by variable-angle spectroscopic ellipsometry analysis is $200 \mathrm{~nm}$. The virgin films were annealed at $135^{\circ} \mathrm{C}$ for $4 \mathrm{~h}$ in air to improve the crystallinity. For polarization-electricfield $(P-E)$ hysteresis loops measurements, Au top electrode was evaporated onto the films through a mask to form an Au-copolymer-Au capacitor structure. The $P-E$ loops measurements were performed using the same method as in Ref. 15. X-ray diffraction measurements were performed on a highly accurate two-axis diffractometer in a Bragg-Brentano geometry using $\mathrm{Cu} K \alpha$ wavelength issued from a $18 \mathrm{~kW}$ rotating anode generator with diffraction angles precision better than $0.002^{\circ}(2 \theta)$.

The thermal cycle performed in this study consists to first cool the as-prepared sample from $300 \mathrm{~K}$ down to $100 \mathrm{~K}$ and then to heat up back to $300 \mathrm{~K}$. Selected ranges of diffraction patterns from $17^{\circ}$ to $21^{\circ}(2 \theta)$ were recorded each $10 \mathrm{~K}$ during 1 h 30 min each. Room temperature X-ray diffraction patterns are shown in Fig. 1. At $300 \mathrm{~K}$, the as-prepared sample (see Fig. 1(a)) exhibits an asymmetric Bragg peak at $19.8^{\circ}$ corresponding to an interplanar spacing of $4.48 \AA$ which indicates that the chains are packed on a hexagonal (or pseudo-hexagonal) lattice of axial length of $5.17 \AA .^{11}$ The asymmetry comes from the intensity of the $\{200\}$ reflection at $19.5^{\circ}$, whose intensity is weaker than that of the $\{110\}$ reflection at around $19.8^{\circ}$. Note that the axial length of the $\beta$ phase lattice in copolymers is larger than that of $\beta$ phase in pure PVDF. This is due to the partial substitution of the hydrogen by the larger fluorine atoms leading to larger unit cell and thus a smaller average dipole moment in copolymers. Surprisingly, as evidenced in Fig. 1(b), the sample having experienced the thermal cycling and back to room temperature displays an additional Bragg peak at $18.4^{\circ}$ corresponding to an interplanar spacing of $4.82 \AA$ contrasting with the situation of the sample without thermal treatment which shows solely the Bragg peak at $19.8^{\circ}$. Such additional 


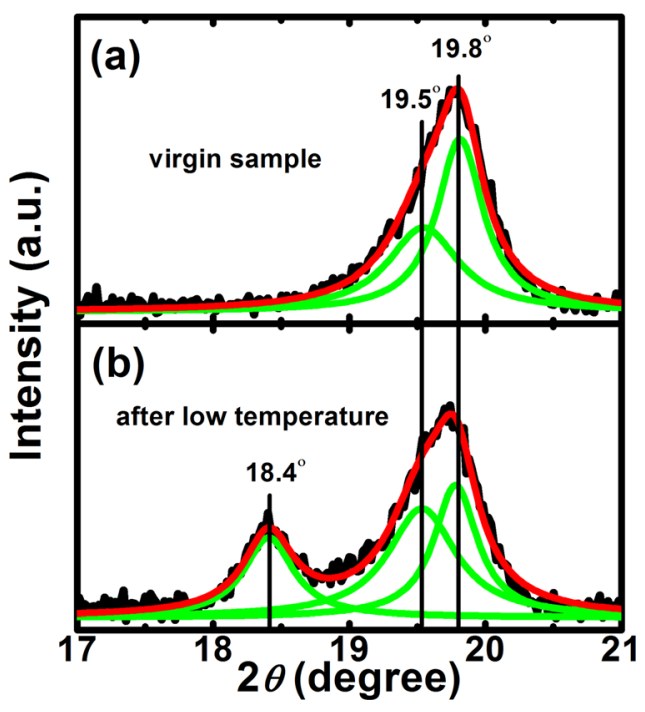

FIG. 1. X-ray diffraction patterns recorded between $17^{\circ}$ and $21^{\circ}$ in $2 \theta$ at 300 K. (a) As-grown sample. (b) Sample having experienced low temperature.

peak may imply a phase transition, though its origin is not clear.

Fig. 2 depicts Bragg peak $2 \theta$ position as a function of temperature in $\mathrm{P}(\mathrm{VDF}-\mathrm{TrFE})$ copolymers during the cooling-heating thermal cycle. The additional peak appears on heating at around $250 \mathrm{~K}$ and its position decreases significantly from $19.5^{\circ}$ at $250 \mathrm{~K}$ down to $18.4^{\circ}$ at $300 \mathrm{~K}$. This transformation is irreversible because the additionally appeared Bragg peak does not disappear after further cooling-heating cycles. It is worth mentioning that strong relaxations also take place at around $250 \mathrm{~K}$ in pure PVDF and copolymers. ${ }^{16-19}$ In this study, we call this relaxation process as $\beta$ relaxation although it is also denoted in the literature, such as $\beta_{a}$ in Ref. 16, $\alpha_{a}$ in Ref. 17, and $\beta_{c}$ in Ref. 19. $\beta$ relaxation has been traditionally assigned to the dynamic glass transition of segments in the amorphous portion of the PVDF polymer and copolymers. ${ }^{17,18,20}$ It has been proposed also that an interphase region, which maintains the conformational characteristics of the crystalline regions, should have a decisive role ${ }^{21}$ in this relaxation process. Indeed, Hahn et al. point out that the $\beta$ relaxation results from the crystal-amorphous interphase in lamellar PVDF crystallites. $^{22}$ The $\beta$ relaxation strength depends linearly on the weight fraction of the PVDF interphase, which indicates that the $\beta$ relaxation may be regarded as a transition of the PVDF

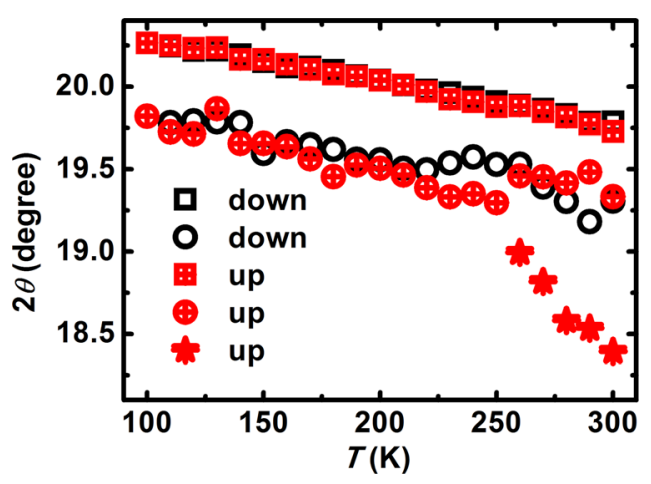

FIG. 2. Bragg peak $2 \theta$ position as a function of temperature from 100 to $300 \mathrm{~K}$ for P(VDF-TrFE) copolymers. interphase. ${ }^{23}$ Moreover, at 230-260 K, both elastic and shear piezoelectric constants associated with chain displacements along the chain axis decrease rapidly while the dielectric constant perpendicular to the dipole moment and chain axis increases. ${ }^{19}$ This indicates the onset at this $\beta$ relaxation temperature of rotational or torsional vibrations of molecular chains and longitudinal motions along the chain. ${ }^{19}$ Besides, the strength of dielectric $\beta$ relaxation decreases noticeably after poling. ${ }^{19}$ Since poling treatment reduces structural defects in crystals, making chain packing in the crystal more regular, the $\beta$ relaxation can be attributed to some structural defects.

Therefore, the chain motions such as rotational and torsional vibrations in the crystal-amorphous interphase are expected to be intense at around $250 \mathrm{~K}$. The crystalamorphous interphase maintains the characteristics of the molecular order of the crystalline region without suffering from the rigid network ${ }^{21}$ restraining the mobility. As a result, the motion activation in the interphase is more energetically favored compared to that in the rigid network of the crystalline regions. Interestingly, this behavior is also consistent with the counterpart of the $\alpha$ relaxation, which stems from molecular motions in the crystalline regions, ${ }^{18,24-26}$ and happens at higher temperatures near the melting temperature $\left(T_{m}\right)$. Actually, $\alpha$ relaxation in $\mathrm{P}(\mathrm{VDF}-\mathrm{TrFE})$ copolymers is related with the ferroelectric to paraelectric transition. ${ }^{16,27}$ It was showed that the $\beta$ and $\alpha$ relaxations arise from the same type of molecular motions, i.e., cooperative trans-gauche transformations at the boundary between all-trans and disordered helical segments. ${ }^{27}$ Similar to the ferroelectric to paraelectric transition occurring at $T_{c}$ in crystalline regions, the change from almost perfect ordered crystal state to less ordered amorphous state may happen within crystalamorphous interphases at $\beta$ relaxation temperature, which therefore leads to the experimental results observed in this work.

In the ferroelectric to paraelectric transition process, copolymers were found to undergo drastic intramolecular changes through introduction of $G$ and $G^{\prime}$ bonds, resulting in an irregular succession of $T G$ and $T G^{\prime}$ groups which are similar to the disordered $3 / 1$ helical conformation of polytrifluoroethylene. $^{28,29}$ Moreover, the paraelectric phase conformation consists of other random combination of transgauche isomers, such as $T_{3} G$ and $T_{3} G^{\prime}$ or even $T_{5} G$ and $T_{5} G^{\prime} \cdot{ }^{11,30,31}$ Long trans sequences unlikely survive above $T_{c}$ as no X-ray reflection characteristics of the trans-planar repetitions were observed. This helical conformation leads to a major extension of the intermolecular distance. The drastic reorganization of the crystalline region through the phase transition has been reported. ${ }^{13}$ In the ferroelectric phase, the coherent crystalline regions appear like flat $(8 \mathrm{~nm})$ platelets of $30 \mathrm{~nm}$-size perpendicular to the chain axis, while in the paraelectric phase the lamellae becomes thinner $(3.5 \mathrm{~nm})$ and bigger $(80 \mathrm{~nm})$. Like ferroelectric to paraelectric transition at $T_{c}$, the change of conformation from all-trans order to helical disorder within crystal-amorphous interphase at around $250 \mathrm{~K}$ is expected to give rise to a lager intermolecular distance which in turn leads to a smaller angle Bragg peak as it appears in the X-ray diffraction pattern shown in Fig. 1. However, it seems that the order-disorder change happening 
at around $250 \mathrm{~K}$ is not due to the introduction of $G$ and $G^{\prime}$ bonds as observed at $T_{c}$ because of potential energy considerations. Indeed, such transition is rather expected to be triggered around defects or boundaries between ordered and disordered phases, which needs less energy. Indeed, shear stresses are introduced by $\operatorname{TrFE}$ units making $G$ or $G^{\prime}$ bonds creation more easily, ${ }^{24}$ while the boundaries need half energy to form disordered structure. It is worth noting that Bernd et al. suggested that the presence of chain defects could result in significant fraction of interphase region in semicrystalline PVDF. ${ }^{22}$ In $\mathrm{P}(\mathrm{VDF}-\mathrm{TrFE})$ copolymers, in addition to the intentionally inserted TrFE units, there are extents of reversed monomeric units, head to head and tail to tail as well as other defects, such as folding chains, kinks, chain ends, and twist chains due to irregular chain sequences. $^{22,32}$ These defects thus influence the interphase and its amount. The defects-filled crystal-amorphous interphase triggering an all-trans order to helical disorder transition due to the chain motions such as abruptly intensive rotational and torsional vibrations is therefore a reasonable mechanism to explain the anomalies observed at $250 \mathrm{~K}$. This interphase transition is also considered in polyethylene and ethylene copolymers. ${ }^{33,34}$ Actually, the formation of interphase in lamellar semicrystalline polymers is required because of the difficulty to abruptly dissipate the order at the crystal surface owing to the chain connectivity. Due to the steric packing problem, the change of the order into more isotropic arrangement in the amorphous state requires significant attrition, as much as $70 \%$, of the chain flux emanating from the crystal surface by reentry of chain sequences to the same lamellae. ${ }^{22,35-37}$ Large amount of interphase or inbetween crystal-amorphous regions exists in copolymers and may explain the additional X-Ray diffraction peak observed in this study [see Fig. 1(a)].

In addition to the interphase, some crystalline regions being defect-rich can also account for the $\beta$ relaxation. Indeed, Takahashi et al. suggested $T G T G^{\prime}$-type kink defects embedded in the all-trans chains, as ‥TTTTTTTTTTGTG' TGTG'TTTTTTTT... ${ }^{24}$ The TrFE units being larger introduce many shear stresses, which can generate $T G T G^{\prime}$ defects in the all-trans chains in crystalline regions. At low temperature, e.g., $100 \mathrm{~K}$ in this study, the smaller intermolecular distances make the influence of shear stress induced by TrFE units stronger and thus favor the amount of $T G T G^{\prime}$ defects. When the sample is heated up, a trans order to helical disorder transition in the neighboring of those defects can take place at $\beta$ relaxation temperature because of the occurrence of abrupt rotational and torsional vibration motions in chains. While further works are required to identify the dominant mechanism of this transition, in both cases, defects affect the dynamic of molecular motions and an ordered to less-ordered state is believed to occur at $\beta$ relaxation temperature.

The order-disorder phase transformation at $\beta$ relaxation temperature may be regarded as a phase transition in somehow similar to that happening in liquid crystals involving partial ordering. The occurrence of this type of transition is supported by the change of the thermal expansion coefficient derived from the additional diffraction peak showed in Fig. 2. Indeed, both phases, i.e., above and below $\beta$ relaxation temperature $T_{\beta}$, with similar hexagonal structure have very different molecular conformations: a disordered 3/1 helical conformation above $T_{\beta}$ and all-trans order below $T_{\beta}$. The thermal expansion coefficient is defined as $\eta=(\partial d / \partial T) / d{ }^{13}$ where $d$ is the lattice parameter. Above $T_{\beta}$, the thermal expansion coefficient is large, $\eta \sim 8 \times 10^{-4} \mathrm{~K}^{-1}$, which is a characteristic feature of disordered structures like glasses, plastic crystals, etc., ${ }^{13}$ while in the more ordered phase below $T_{\beta}$, it is only $1.36 \times 10^{-4} \mathrm{~K}^{-1}$.

To further support our viewpoint, $P-E$ loops are measured on heating from $180 \mathrm{~K}$ to $300 \mathrm{~K}$. As showed in Fig. 3 (a), nearly rectangular $P-E$ loops are obtained whatever the temperature attesting of the presence of a ferroelectric state within the whole investigated range of temperatures. The coercive electric field $\left(E_{c}\right)$ as well as the remnant polarization $\left(P_{r}\right)$ decrease with temperature increasing [Fig. 3(a)], which indicates that polarization switching is thermally activated. ${ }^{10}$ In case of any partial loss of polar order in the films at $\beta$ relaxation temperature, there must be some traces in the temperature dependence of $P_{r}$ and $E_{c}$. Indeed, as shown in Fig. 3(b), both temperature dependence of $P_{r}$ and $E_{c}$ change their slope at $T_{\beta} \sim 250 \mathrm{~K}$. On heating above $T_{\beta}, P_{r}$ starts to decrease faster meaning that the global phase becomes less polar. At the same time, $E_{c}$ decrease slows down above $T_{\beta}$ showing that it becomes more difficult to switch the polarization state. These observations are consistent with the fact that at $T_{\beta} \sim 250 \mathrm{~K}$ the ordered to less ordered phase transition only occurs in some regions of the films that could be either at the interphase and/or in defect-filled crystalline regions. Above the partial ordering/disordering transition temperature $T_{\beta}$, the sample remains ferroelectric. However, the relative ratio of polar disorder in respect to polar order becomes smaller because of the order-disorder change expected to occur in the interphase and/or defect-rich crystalline regions. As a matter of fact, this change causes $P_{r}$ and $E_{c}$ to decrease faster and slower, respectively, on increasing temperature.

In summary, as-grown $\mathrm{P}(\mathrm{VDF}-\mathrm{TrFE})$ copolymers films underwent an irreversible partial order-disorder transformation at $T_{\beta} \sim 250 \mathrm{~K}$ after being preliminarily cooled down to $100 \mathrm{~K}$. This transition is evidenced by the appearance of an additional X-Ray diffraction peak at around $250 \mathrm{~K}$ which is concomitant to the so-called $\beta$ relaxation temperature associated with change of the system dynamic. This orderdisorder-like transformation is attributed to an all-trans order to helical disorder transition. It has been suggested to occur in defect-rich regions like crystal-amorphous interphases and/or crystalline areas associated to randomly distributed
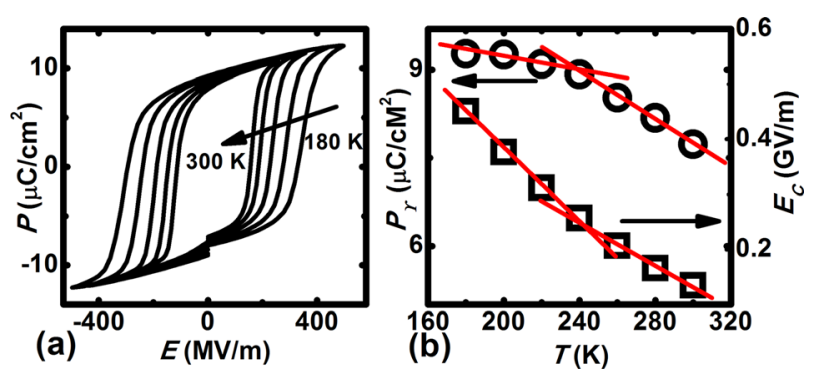

FIG. 3. Temperature dependence of (a) $P-E$ loops, and (b) remnant polarization $\left(P_{r}\right)$ (left), coercive electric field $E_{c}$ derived from $P-E$ loop (right) of $\mathrm{P}(\mathrm{VDF}-\mathrm{TrFE})$ films. The straight lines are guide to the eyes. 
TrFE units. Such defects cause intensive chain motions such as rotational and torsional vibrations at $\beta$ relaxation temperature. This interpretation is supported by the temperature dependence of the expansion coefficient as well as the ferroelectric parameters, i.e., $P_{r}$ and $E_{c}$.

This work was supported by the Major State Basic Research Development Program (Grant No. 2013CB922302), the Natural Science Foundation of China (Grant Nos. 11374320 and 11104301), and Natural Science Foundation of Shanghai (13JC1406000 and 14JC1406500). B.B.T., X.F.B., Y.L., and B.D. would also wish to acknowledge the China Scholarship Council (CSC).

${ }^{1}$ R. G. Kepler and R. A. Anderson, J. Appl. Phys. 49, 1232 (1978).

${ }^{2}$ T. Furukawa, M. Date, M. Ohuchi, and A. Chiba, J. Appl. Phys. 56, 1481 (1984).

${ }^{3}$ T. Furukawa, Phase Transitions 18, 143 (1989).

${ }^{4}$ Y. C. Yuan, T. Yin, M. Z. Rong, and M. Q. Zhang, eXPRESS Polym. Lett. 2, 238 (2008).

${ }^{5}$ S. R. Forrest, Nature 428, 911 (2004).

${ }^{6}$ G. H. Gelinck, H. E. A. Huitema, E. van Veenendaal, E. Cantatore, L. Schrijnemakers, J. B. P. H. van der Putten, T. C. T. Geuns, M. Beenhakkers, J. B. Giesbers, B. Huisman, E. J. Meijer, E. M. Benito, F. J. Touwslager, A. W. Marsman, B. J. E. van Rens, and D. M. de Leeuw, Nat. Mater. 3, 106 (2004).

${ }^{7}$ E. Cantatore, T. C. T. Geuns, G. H. Gelinck, E. van Veenendaal, A. F. Gruijthuijsen, L. Schrijnemakers, S. Drews, and D. M. de Leeuw, IEEE J. Solid-State Circuits 42, 84 (2007).

${ }^{8}$ T. Levard, P. J. Diglio, S.-G. Lu, C. D. Rahn, and Q. M. Zhang, Smart Mater. Struct. 21, 012001 (2012).

${ }^{9}$ T. Yagi, M. Tatemoto, and J. Sako, Polym. J. 12(4), 209 (1980).

${ }^{10}$ B. B. Tian, Z. H. Chen, A. Q. Jiang, X. L. Zhao, B. L. Liu, J. L. Wang, L. Han, Sh. Sun, J. L. Sun, X. J. Meng, and J. H. Chu, Appl. Phys. Lett. 103, 042909 (2013).
${ }^{11}$ A. J. Lovinger, T. Furukawa, G. T. Davis, and M. G. Broadhurst, Polymer 24, 1225 (1983).

${ }^{12}$ K. Tashiro, K. Takano, M. Kobayashi, Y. Chatani, and H. Tadokoro, Ferroelectrics 57, 297 (1984).

${ }^{13}$ J. F. Legrand, Ferroelectrics 91, 303 (1989).

${ }^{14}$ J. Petzelt, J. F. Legrand, S. Pacesova, S. Kamba, G. V. Kozlov, and A. A. Volkov, Phase Transitions 12, 305 (1988).

${ }^{15}$ B. B. Tian, X. L. Zhao, B. L. Liu, J. L. Wang, Li. Han, J. L. Sun, X. J. Meng, and J. H. Chu, Appl. Phys. Lett. 102, 072906 (2013).

${ }^{16}$ S. H. Zhang, R. J. Klein, K. L. Ren, B. J. Chu, X. Zhang, and J. Runt, J. Mater. Sci. 41, 271 (2006).

${ }^{17}$ H. Sasabe, S. Saito, M. Asabina, and H. Kakutani, J. Polym. Sci. Part A-2 7, 1405 (1969).

${ }^{18}$ S. Yano, J. Polym. Sci. Part A-2 8, 1057 (1970).

${ }^{19}$ K. Omote, H. Ohigashi, and K. Koga, J. Appl. Phys. 81, 2760 (1997).

${ }^{20}$ N. Koizumi and S. Yano, J. Polym, Sci. B: Polym. Lett. 7, 59 (1969).

${ }^{21}$ R. Gregorio, Jr. and E. M. Ueno, J. Mater. Sci. 34, 4489 (1999).

${ }^{22}$ B. Hahn, J. Wendorff, and D. Y. Yoon, Macromolecules 18, 718 (1985).

${ }^{23}$ Y. Ando, T. Hanada, and K. Saitohj, J. Polym. Sci. B: Polym. Lett. 32, 179 (1994).

${ }^{24}$ Y. Hirata and T. Kotaka, Polym. J. 13, 273 (1981).

${ }^{25}$ D. R. Paul and J. O. Altamirano, Adv. Chem. Ser. 142, 371 (1975).

${ }^{26}$ Z. H. Liu, P. Marechal, and R. Jerome, Polymer 38(19), 4925 (1997).

${ }^{27}$ T. Furukawa, M. Ohuchi, A. Chiba, and M. Datela, Macromolecules 17, 1384 (1984).

${ }^{28}$ G. T. Davis, T. Fumkawa, A. J. Lovinger, and M. G. Broadhurst, Macromolecules 15, 329 (1982).

${ }^{29}$ R. R. Kolda and J. B. Lando, J. Macromol, Sci. B: Phys. 11, 21 (1975).

${ }^{30}$ K. Tashiro, K. Takano, M. Kobayashi, Y. Chatani, and H. Tadokoro, Polymer 25, 195 (1984).

${ }^{31}$ S. Green, J. P. Rabe, and J. F. Rabolt, Macromolecules 19, 1725 (1986).

${ }^{32}$ R. Hasegawa, Y. Takahashi, Y. Chatani, and H. Tadokoro, Polym. J. 3, 600 (1972).

${ }^{33}$ R. Popli and L. Mandelkern, Polym. Bull. 9, 260 (1983).

${ }^{34}$ R. Popli, M. Glotin, L. Mandelkern, and R. S. Benson, J. Polym. Sci., Polym. Phys. Ed. 22, 407 (1984).

${ }^{35}$ P. J. Flory, J. Am. Chem. Soc. 84, 2857 (1962).

${ }^{36}$ P. J. Flory, D. Y. Yoon, and K. Dill, Macromolecules 17, 862 (1984).

${ }^{37}$ D. Y. Yoon and P. J. Flory, Macromolecules 17, 868 (1984). 